$11-2006$

\title{
Procedure to Quantify Biofilm Activity in Carriers Used in Wastewater Treatment Systems
}

\author{
James Bolton \\ Kinetico Inc. \\ Archana Tummala \\ Cleveland State University \\ Chirag Kapadia \\ Cleveland State University \\ Manoj Dandamudi \\ Cleveland State University
}

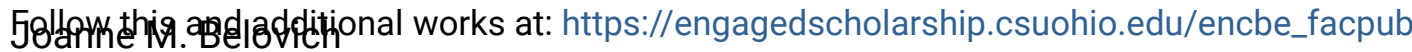

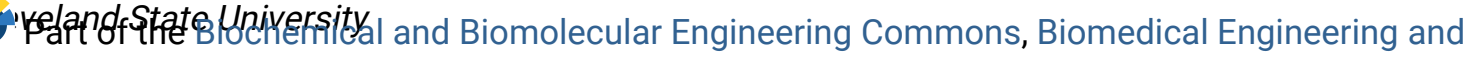

Bioengineering Commons, and the Environmental Engineering Commons

How does access to this work benefit you? Let us know!

\section{Original Citation}

Procedure to Quantify Biofilm Activity on Carriers Used in Wastewater Treatment Systems Bolton, J., Tummala, A., Kapadia, C., Dandamudi, M., and Belovich, J. Journal of Environmental Engineering 2006 $132: 11,1422-1430$

\section{Repository Citation}

Bolton, James; Tummala, Archana; Kapadia, Chirag; Dandamudi, Manoj; and Belovich, Joanne M., "Procedure to Quantify Biofilm Activity in Carriers Used in Wastewater Treatment Systems" (2006). Chemical \& Biomedical Engineering Faculty Publications. 30.

https://engagedscholarship.csuohio.edu/encbe_facpub/30

This Article is brought to you for free and open access by the Chemical \& Biomedical Engineering Department at EngagedScholarship@CSU. It has been accepted for inclusion in Chemical \& Biomedical Engineering Faculty Publications by an authorized administrator of EngagedScholarship@CSU. For more information, please contact library.es@csuohio.edu. 


\title{
PROCEDURE TO QUANTIFY BIOFILM ACTIVITY IN CARRIERS USED IN WASTEWATER TREATMENT SYSTEMS
}

\author{
James Bolton, Kinetico Inc. \\ Archana Tummala, Chirag Kapadia, \\ Manoj Dandamudi, and Joanne M. Belovich, Cleveland State University
}

\begin{abstract}
A procedure is presented for evaluating and comparing the biological activity of biofilms attached to various biofilm carriers by measurement of the glucose consumption rate. This technique allows for the economical design and selection of small particulate biofilm carriers that will maximize substrate removal when used in industrial-scale fluidized bioreactors. Methods for ensuring reproducible results are described. To support the glucose consumption rate findings, biofilm dry weights were obtained at the conclusion of activity rate experiments, and scanning electron micrographs were taken to evaluate the presence of biofilm and to view surface characteristics. Fourteen different biofilm carriers were evaluated ranging from commercially available products to novel carriers designed specifically for this study. Carriers that exhibited the highest reaction rates in descending order included: Syntrex 1220 (Kinetico, Inc.), Kaldnes Carrier Element-Modified (Kaldnes North America, Inc.), Kaldnes Carrier Element Original (Kaldnes North America, Inc.), Macrolite Modified CEPP-02 (Kinetico, Inc.), Macrolite 357 (Kinetco, Inc), and Virgin Foam Cubes (BB Bradley Co.). Results showed that the accumulation of biofilm depended most strongly on carrier surface properties, such as surface roughness and specific surface area. The biofilm activity as measured by glucose consumption rate correlated well with activity determinations made by COD measurements when a complex carbohydrate was used as substrate in place of glucose. Substrate consumption rates in microreactors were within $\pm 43 \%$ of those measured in a 3-L bioreactor. The method presented here produced highly reproducible results and may be used to accurately and economically screen a large number of newly-designed carriers for application in industrial bioreactor processes.
\end{abstract}

\section{Introduction}

Recent bioreactor innovations in wastewater treatment systems have included the fluidized bed, moving bed, and biological aerated filter reactor designs [Rodgers and Zhan 2004; Münch et al. 2000; Ødegaard 2000; Sanyal, "Process and apparatus for removal of organic pollutants from waste water," U.S. Patent No. 5,217,616 (1997); Lodaya, "Process for biochemical oxidation of ultra-toxic wastewater in a packed bed bioreactor," U.S. Patent No. 5,403,487 (1995); Sato, "Carrier for bioreactor and method of treating wastewater using carrier," European Patent No. 0849228A2 (1997)]. These reactors employ small granular particles ("carriers") that are used as solid support for microbial at-

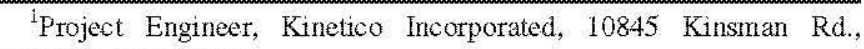
Newbury, OH 44065.

${ }^{2}$ Graduate Student, Dept. of Chemical and Biomedical Engineering, Cleveland State Univ, Cleveland, OH 44115-2425.

${ }^{2}$ Graduate Student, Dept. of Chemical and Biomedical Engineering, Cleveland State Univ, Cleveland, OH 44115-2425.

${ }^{4}$ Graduate Student, Dept. of Chemical and Biomedical Engineering, Cleveland State Univ., Cleveland, OH 44115-2425.

${ }^{5}$ Associate Professor, Dept. of Chemical and Biomedical Engineering, Cleveland State Univ., Cleveland, OH 44115-2425 (comesponding author).

Note. Discussion open until April 1, 2007. Separate discussions must be submitted for individual papers. To extend the closing date by one month, a written request must be filed with the ASCE Managing Editor. The manuscript for this paper was submitted for teview and possible publication on December 30, 2004; approved on May 15, 2006. This paper is part of the Journal of Environmental Engineering. Vol. 132, No. 11, November 1, 2006. OASCE, ISSN 0733-9372/2006/11-1422-$1430 / \$ 25.00$. tachment ("biofilm"). Biofilm carriers are found in a variety of shapes, sizes, and materials, and significant effort has been made to optimize these carriers for improved biological activity [Trubenbach, "Monomodal and polymodal catalyst supports and catalysts having narrow pore size distributions and their production," U.S. Patent No. 5,935,898 (1999)]. Commercial examples of these processes include the Kaldnes Moving Bed Biofilm Reactor (Kalnes North America, Inc, Providence, R.I.), OndeoDegremont Biofor Biological Filtration Process (Infilco Degremont, Inc., Richmond, Va.), Envirogen Fluidized Bed Reactor (Envirogen, Lawrenceville, N.J.), Severn Trent Tetra Submerged Aerated Filter (Severn Trent Services, Fort Washington, Pa), and the US Filter Biostyr Process (Siemens, Warrendale, Pa.). The effectiveness of such processes depends on appropriate processing conditions for the biofilm carriers, including efficient transport of substrate and growth elements to the biofilm and maintenance of an effective biofilm thickness. Beyond such reactor design components, another important factor in process efficiency is the efficacy of the biofilm carrier itself.

Performance of a wastewater treatment process is typically evaluated using analytical measurements such as biochernical oxygen demand (BOD), chemical oxygen demand (COD), and total organic carbon (TOC). These measurements provide information on the macroscopic performance of specific bioreactor processes, but provide limited quantitative information on cellular biological activity.

A variety of academic research has been conducted on the mechanisms of biofilm adhesion to solid surfaces, and the correlation of adhesion to various characteristics of the carriers such as surface energy, hydrophobicity, surface charge, roughness, and pore size. Other work has focused on the morphology and physics 
of the biofilm itself (Fletcher 1991; Becker 1998; Jones et al. 1996; Bos et al. 2000; Flint et al. 1997; Teixeira and Oliveira 1998; Van Loosdrecht et al. 1995). A variety of tools have been used to study and quantify microbial proliferation, such as light fluorescence (Jones et al. 1996), fluorescence in situ hybridization (Araki et al. 1999; Davey and O'Toole 2000; Wimpentry et al. 2000), scanning electron microscopy (Brown et al. 1998), transmission electron microscopy (Sanford et al. 1995), X-ray microscopy (Gilbert et al. 1999), and confocal laser scanning microscopy (Wimpenny et al. 2000), and sensor triangulation (Okkerse et al. 2000). There are also myriad other biochemical parameters that can be measured and directly inferred to biofilm activity (Lazarova and Manem 1995). These techniques, although very insightful to the ecology of biofilm behavior, are limited because of cost, complexity, and the invasive disruption of intra- and extracellular processes. Moreover, the correlation between substrate removal rate and one of the biofilm descriptors (e.g., ATP, protein, or DNA content, film thickness) is not always reliable (Lazarova and Manem 1995). These methods may not provide practical assistance in the design and selection of biofilm carriers for fullscale reactor design.

Other techniques commonly employed to measure biological activity include oxygen consumption, carbon dioxide production, and substrate consumption rates. The most reliable quantitative tool is the direct measurement of in situ substrate removal rate using the fluidized carriers. There is limited literature available on the biological activity of biofilms adhered to fluidized carriers. Literature that is available on this topic includes several laboratory and pilot scale studies on materials such as sintered beads (Salkinoja-Salonen et al. 1983); red drain clay, sand, and a variety of plastics (Shreve et al. 1991); polyurethane foam cubes (Chandravathanam and Murthy 1999); low density granular polypropylene (Yoo and Kim 2001); floating polyethylene (Belgiomo et al. 2003); low-density granular polystyrene (Payraudeau et al. 2001); and sintered glass (Breitenbucher et al. 1990).

Most current industrial methods for testing biofilm growth on engineered materials involves flat samples placed in flowing streams. Evaluation of fluidized granules is less common. There is a need to standardize a small-scale laboratory procedure that quickly and economically evaluates numerous small solid particulates for their efficacy as biofilm carriers in industrial reactors. This systern must have many of the characteristics of the industrial process, such as the fluidized environment and mixed cell culture, while being at a small scale so that numerous evaluations can be conducted simultaneously.

This paper describes in detail an experimental process to evaluate whether one carrier possesses a distinct advantage over another carrier in terms of biofilm activity, given all other conditions remain constant. Potential pitfalls in experimental design and data analysis are highlighted. The length of time needed to establish a steady-state biofilm activity and influence of sampling time and wall growth on activity measurements were determined. The validity of 0 th order reaction kinetics to represent substrate consumption rate was presented. This procedure was used to compare biofilm activity on a number of custom-designed carriers against several commercially available products.

Carrier efficacy was measured by the biological activity of the biofilm adhered to each carrier, with biological activity measured in terms of substrate removal rate. Glucose was selected as the sole substrate and energy source because it is easily prepared in reproducible batches, can be utilized by a wide variety of important microorganisms, and the glucose concentrations can be measured quickly, economically, and in small samples. The glucose consumption rate does not provide detailed biological information, such as the structure or type of biofilm present; however, it does provide a measure of the overall metabolic activity of the microorganisms attached to different biofilm carriers. By providing identical reactor and envirommental conditions, the relative biological activity between carriers can be assessed.

The procedure utilized a standardized and diverse heterotrophic culture of microorganisms and plenty of opportunity was provided to cross contaminate the microorganisms between reactors and batch experiments. The researchers attempted to create an identical biofilm ecology between each carrier to eliminate this variable as a reason for varying substrate removal rates. In order to measure substrate removal from the biofilm attached to the carriers only, procedures were adopted that minimized the growth of biofilm on other surfaces within the microreactor. Thorough mixing and aeration was provided to ensure that substrate removal was not limited by mass transport or bulk oxygen concentration. Nutrients were provided in excess and all attempts were made to ensure that environmental conditions did not limit substrate removal.

The majority of experiments were conducted in small flasks, described as microreactors, using glucose as a simple substrate. To evaluate the robustness and scalability of this procedure several additional experiments were conducted. The relevance of the glucose uptake rate measurement as a measure of biofilm activity in a complex-carbohydrate wastewater stream, as exemplified by waste from a food or paper processing plant, was evaluated by repeating the microreactor experiments using molasses as the more complex substrate. Molasses reduction rate was measured using the COD measurement. The influence of scale-up on substrate uptake rate was evaluated by conducting additional experiments using a bench-scale bioreactor with glucose as the substrate.

\section{Materials and Methods}

Fourteen different biofilm carriers were evaluated during this study. The carriers are summarized in Table 1 and are sorted by those that are commercially available and those that were novel for this study. The choice of carriers was based on many factors including surface properties, composition, surface area, particle shape, commercial availability, and scale-up bioreactor economics.

The biological activity test consisted of three separate procedures: (1) inoculation of each biofilm carrier; (2) growth of the biofilm; and (3) measurement of the substrate uptake rate.

\section{Inoculation}

The inoculum was prepared from Polyseed (Integrated Biochemical Systems, Inc.), which contains a diverse and standardized culture of heterotrophic microorganisms free of human pathogens. The media was Bacto Bushnell-Haas broth (Difco Laboratories) supplemented with either a glucose stock solution or a molasses stock solution for a final sugar concentration of $1.0 \mathrm{~g} / \mathrm{L}$, which resulted in a COD of $1.0 \mathrm{~g} / \mathrm{L}$. The glucose and molasses stock solutions were prepared with either Reagent Grade D \pm Glucose (Sigma) or food-grade molasses at $300 \mathrm{~g} / \mathrm{L}$ and filtered through $0.2 \mu \mathrm{m}$ membrane and maintained under sterile conditions. One Polyseed capsule was added to $850 \mathrm{~mL}$ of media in an Erlenmeyer flask. An aeration rod was used to mix and provide dissolved 
Table 1. Biofilm Carriers

\begin{tabular}{|c|c|c|}
\hline Carriers & Manufacturer & Description of carrier; effective diameter (ED) \\
\hline \multicolumn{3}{|l|}{ Commercially available biofilm carriers } \\
\hline Kaldnes Carrier Element_Original (KA-O) & Kaldnes North America, Inc. & $\begin{array}{l}\text { Honeycomb shaped polyethylene particle with multiple fins } \\
\text { located externally - smooth surface; } E D=10 \mathrm{~mm}\end{array}$ \\
\hline Kaldnes Carrier Element-Modified (KA-M) & Kaldnes North America, lnc. & $\begin{array}{l}\text { Similar to KA-O with multiple fins located intemally- } \\
\text { smooth sheltered surface; } E D=10 \mathrm{~mm}\end{array}$ \\
\hline Waterline Pellet (WL) & Alchem Industries & $\begin{array}{l}\text { Oblong shaped recycled plastic beads with irregular groves } \\
\text { stamped on two surfaces-smooth surface; } E D=5 \mathrm{~mm}\end{array}$ \\
\hline Virgin Polypropylene (VPP) & M. Holland Co. & $\begin{array}{l}\text { Polypropylene plastic beads-very smooth hard surface; } \\
\mathrm{ED}=4 \mathrm{~mm}\end{array}$ \\
\hline Virgin Foam Cubes (VFC) & BB Bradley Co. & $\begin{array}{l}\text { High-density polypropylene foam cubes with a regular shaped } \\
\text { porous surface-coarse surface; } \mathrm{ED}=6 \mathrm{~mm}\end{array}$ \\
\hline Macrolite 357 (ML357) & Kinetico, Inc. & $\begin{array}{l}\text { Ceramic sphere with a porous rough surface-course surface; } \\
\mathrm{ED}=4 \mathrm{~mm}\end{array}$ \\
\hline \multicolumn{3}{|l|}{ Novel carriers for this study } \\
\hline Syntrex 1220 (SY1220) & Kinetico, Inc. & $\begin{array}{l}\text { Extremely buoyant spherical particle consisting of a phenolic } \\
\text { plastic/glass bubble shell-coarse surface; } \mathrm{ED}=1.25 \mathrm{~mm}\end{array}$ \\
\hline Recycled Plastic (RP) & Kinetico, Inc. & $\begin{array}{l}\text { Recycled plastic ground into a variety of shapes - smooth } \\
\text { surface; } E D=4 \mathrm{~mm}\end{array}$ \\
\hline Macrolite Modified Foam Cubes (MLFC) & Kinetico, Inc. & VFC modified with Macrolite_coarse surface; $\mathrm{ED}=5 \mathrm{~mm}$ \\
\hline Extruded and Chopped Polypropylene (CPP) & Kinetico, Inc. & $\begin{array}{l}\text { Chip made of extruded polypropylene and chopped into } \\
\text { regular shapes-smooth surface; } \mathrm{ED}=4 \mathrm{~mm}\end{array}$ \\
\hline Chopped Expanded Polypropylene-Low (CEPP-02) & Kinetico, Inc. & $\begin{array}{l}\text { Minimally expanded polypropylene chip-smooth surface } \\
\text { with some pores; } \mathrm{ED}=4 \mathrm{~mm}\end{array}$ \\
\hline Chopped Expanded Polypropylene-Medium (CEPP-04) & Kinetico, Inc. & $\begin{array}{l}\text { Moderately expanded polypropylene chip—smooth surface } \\
\text { with pores; } \mathrm{ED}=4 \mathrm{~mm}\end{array}$ \\
\hline Chopped Expanded Polypropylene-High (CEPP-06) & Kinetico, Inc. & $\begin{array}{l}\text { Highly expanded polypropylene chip—smooth surface with } \\
\text { many pores; } E D=4 \mathrm{~mm}\end{array}$ \\
\hline Macrolite Modified CEPP-02 (MLCEPP-02) & Kinetico, Inc. & $\begin{array}{l}\text { CEPP- } 02 \text { modified with Macrolite to increase surface } \\
\text { roughness-coarse surface; } E D=4 \mathrm{~mm}\end{array}$ \\
\hline
\end{tabular}

oxygen to the solution. Gentle aeration was maintained for $60 \mathrm{~min}$ to activate the dehydrated microorganisms.

\section{Biofilm Growth}

The biofilm was cultivated on the surface of the carriers in 125-mL widemouth Erlenmeyer flasks (called the microreactors). The microreactors contained $50 \mathrm{~mL}$ of liquid media and $10 \mathrm{~mL}$ bulk volume of solid carrier. Each carrier was tested in triplicate. Up to 16 microreactors were kept at constant temperature at $30 \pm 1^{\circ} \mathrm{C}$ and mixed at $156 \mathrm{rpm}$ in an Orbit Shaker Bath Model 3540. The carrier densities were all slightly less than that of water, enabling them to be kept easily in suspension. Each flask was aerated by both a shaking motion and by air sparging. Sparging occurred with filtered air supplied through an aeration rod containing a high-density porous plastic frit. A low-pressure gas manifold connected to a panel of rotometers and control valves was designed to apply the same amount of aeration to each flask (0.4-0.6 Lpm per flask) (Fig. 1). This strategy enabled the operation of 16 microreactors simultaneously, each subject to nearly identical environmental conditions. Dissolved oxygen (DO) concentrations were measured in the flasks periodically (WTW Multl-350i oxygen probe) to verify that the DO was above $50 \%$

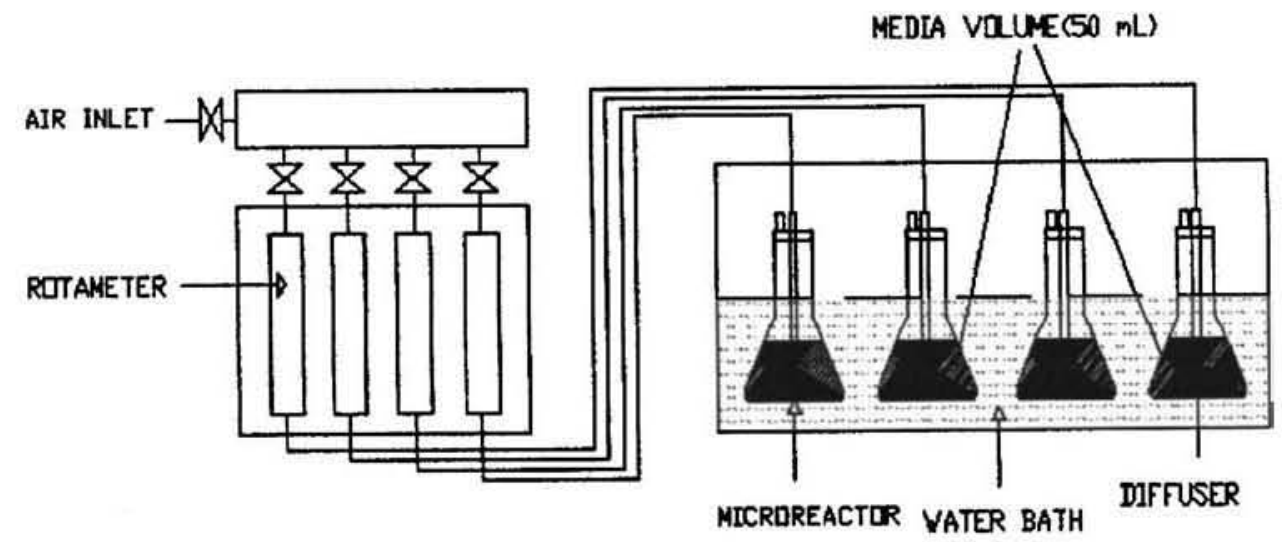

Fig. 1. Microreactor setup 
saturation. The microreactors were sampled once daily and either the glucose concentration (YSI 2700 D Select Biochemistry Analyzer) or the COD (when using the molasses media) (Model 45600 , Hach Co.) and $\mathrm{pH}$ (Orion) were measured. Samples were filtered through a $0.2-\mu m$ membrane prior to COD measurement. COD values were found to correlate directly with substrate concentrations (both for glucose and molasses) and thus are reported in equivalent carbohydrate concentrations. When the substrate concentration dropped to zero a sufficient amount of glucose or molasses stock solution was added to the existing liquid in each microreactor to bring the substrate concentration up to approximately $1.0 \mathrm{~g} / \mathrm{L}$. When the $\mathrm{pH}$ dropped to inhibitory levels $(<5.0)$, it was assumed that the surfaces of the biofilm carriers were partially colonized and the liquid broth was changed. The first media change occurred two to three days after inoculation. Complete biofilm colonization was then encouraged over a period of three to four weeks. The flasks were not changed during this period.

\section{Substrate Consumption Rate Measurement}

Following the biofilm development phase, the carriers were rinsed with fresh broth solution and transferred to clean and sterile microreactors fitted with clean and sterile aeration rods. Each microreactor was then "fed" almost simultaneously by adding $50 \mathrm{~mL}$ of $1.0 \mathrm{~g} / \mathrm{L}$ broth solution using a glass syringe and placed in the shaker bath at the same conditions as described above. Samples of broth were withdrawn from each microreactor periodically and analyzed for glucose concentration or COD.

Separate experiments were conducted to quantify the effect of biofilm wall growth on substrate consumption rate measurement. To eliminate biofilm growth on surfaces other than the carrier, additional cleaning, mixing, and rinsing procedures were adopted during this phase of the procedure. Cleaning of the microreactors prior to activity testing involved a wash in a detergent solution, followed by a thorough rinse and occasionally a soak in $10 \%$ sulfuric acid, and then a $30 \mathrm{~min}$ autoclave at $130^{\circ} \mathrm{C}$. The aeration. rods were washed in detergent solution, followed by soaking in $1 \%$ hydrochloric acid, then soaking in $1 \%$ sodium hydroxide, and completed by multiple rinses with. DI water. To remove suspended microorganisms located within interstitial fluid pockets, and to ensure uniform cropping of biofilm thickness, the biofilm carriers were transferred to a clean microreactor and placed on a vortex mixer for $10 \mathrm{~s}$ (Scientific Industries Gene 2 Model G-560, number 8 setting). The microreactors were drained using a vacuum apparatus, filled with $50 \mathrm{~mL}$ of glucose-free Bushnell-Haas broth, and mixed again and drained.

\section{Scale-up Testing}

To evaluate the scalability of the microreactor procedure, additional testing was conducted in a 3 - $\mathrm{L}$ working volume glass reactor with an internal diameter of $13.5 \mathrm{~cm}$ and a height of $30 \mathrm{~cm}$. Air was supplied via diffusers at flow rates around $3 \mathrm{Lpm}$. DO concentration was periodically monitored and the air supply adjusted to maintain the $\mathrm{DO}$ above $4.5 \mathrm{mgO}_{2} / \mathrm{L}$. The temperature was maintained at $30 \pm 1^{\circ} \mathrm{C}$ using a themocouple and heating tape. Disinfection and cleaning procedures were performed for glassware and carriers as discussed previously for the microreactor experiment. Biofilm inoculation and growth were conducted similarly to that described for the microreactor experiments. Following the three-week biofilm development procedure, after an overnight fast the stock solution was added to the reactor. After $1 \mathrm{~h}$, the substrate consumption rates were measured.

\section{Data Analysis}

The substrate consumption rate was calculated from the glucose or COD profiles, assuming 0th order kinetics (to be validated in "Results" section), using the following model

$$
\frac{d C}{d t}=-k
$$

where $C=$ substrate concentration; and $k=$ substrate consumption rate (e.g., the rate constant). Assuming $k$ is constant, and integrating Eq. (1) yields a linear decrease in substrate concentration with time

$$
C-C_{0}=-k t
$$

Data from each triplicate set were combined into one data set, providing 15-18 data points for analysis. The rate constant $k$ and percent errors $(95 \%$ confidence interval) were calculated from the slope of the data using linear least squares regression. The rate constant with the minimum percentage error was calculated by progressively eliminating the latest data points in time for every kinetics experiment for each carrier. This process eliminated the data points at the end of the experiment, as the glucose concentrations approached zero, at which point the system clearly becomes limited by substrate supply. A minimum of twelve points were used to calculate the rate constant for each experiment. The substrate consumption rate $K$, based on carrier dry bulk volume, was calculated by multiplying $k$ by the ratio of broth volume/ carrier bulk volume.

\section{Analytical Procedures}

Biofilm dry weights were obtained at the conclusion of the activity rate experiments. The carriers were rinsed with glucose-free Bushnell-Haas broth solution and dried ovemight in an oven at $50^{\circ} \mathrm{C}$. The weights of the dried carriers were measured. The carriers were soaked for $24 \mathrm{~h}$ in $10 \%$ Minncare solution (Minntech Corp., Minneapolis), then rinsed with deionized (DI) water and dried overnight at $50^{\circ} \mathrm{C}$ and weighed. This process was repeated using a stronger Minncare solution $(20 \%)$ until negligible weight loss occurred. The difference between the initial and final weight for each carrier resulted in the biofilm dry weight measurement.

Scanning electron microscope (SEM) pictures of carriers were taken before and after growth of the biofilm. The carriers were fixed with $2 \%$ glutaraldehyde, dehydrated with $50 \%$ ethanol in water solution for $15 \mathrm{~min}$, followed by dehydrations in 70, 80, 90, 95 , and $100 \%$ alcohol solutions. This was followed by critical point drying using hexamethyldisilazane (HMDS, Ted Pella, Inc.). The carriers were coated with gold and then SEM micrographs were taken (Nation 1983).

The surface areas of select carriers were measured using either a mercury porosimeter (Micromeritics), at 30,100, and 180 psia or nitrogen adsorption (Micromeritics).

\section{Results}

The growth of biofilm on the carriers was confirmed with select SEM images of carriers before inoculation and following four weeks of biofilm development (see Fig. 2 for sample images of MLCEPP-04 carriers). After three to four weeks of biofilm devel- 


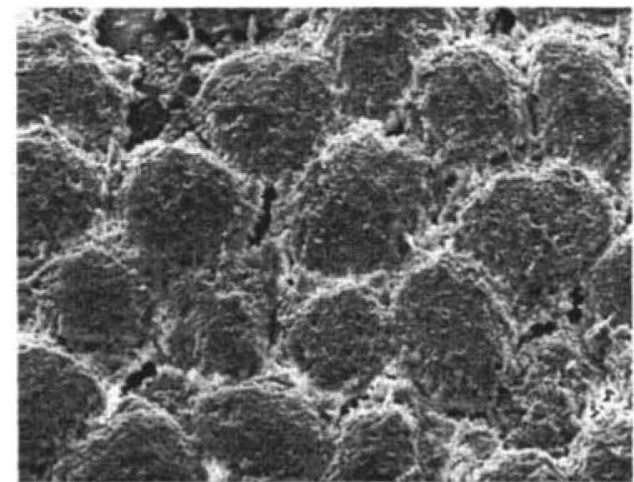

$$
\left[\frac{100 \mu \mathrm{m}}{10}\right.
$$

(a)

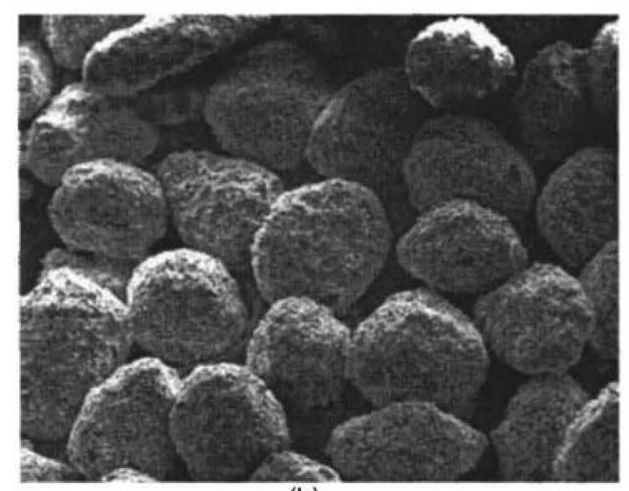

(b)

Fig. 2. SEM pictures of MLCEPP-04 carriers at $100 \mathrm{X}$ magnification (each "spheroid" is a particle of Macrolite fines on the surface of the carrier): (a) with approximately four weeks growth of biofilm; (b) without biofilm

opment, the substrate consumption rates were measured. A sample data set with KA-O carriers shown in Fig. 3 confirms the validity of the 0th order kinetics model, up to $100 \mathrm{~min}$. Concentration profiles among each triplicate set were similar. The DO profile measured during a microreactor glucose consumption rate test with ML 357 carriers is shown in Fig. 4, showing that the bulk oxygen concentration remained above $50 \%$ saturation. Periodic measurements in other microreactors verified that DO was at or above $50 \%$ (data not shown).

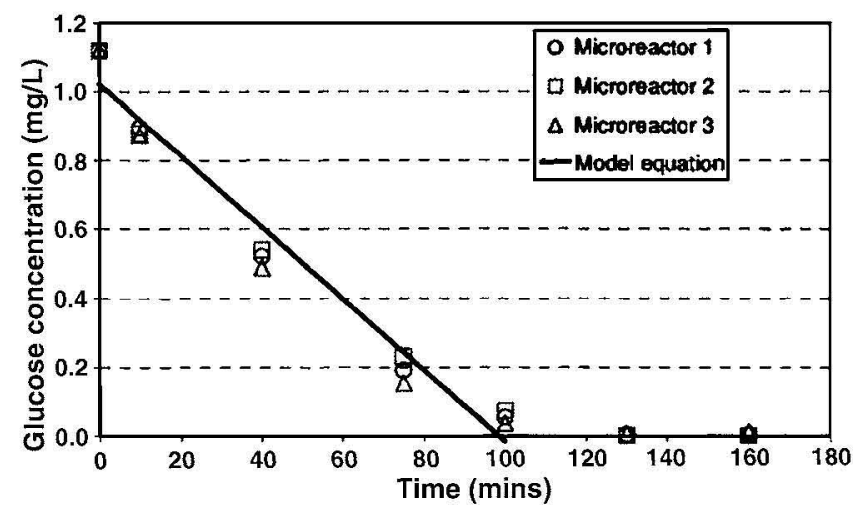

Fig. 3. Glucose concentration profiles from three microreactors using the KA-O carrier

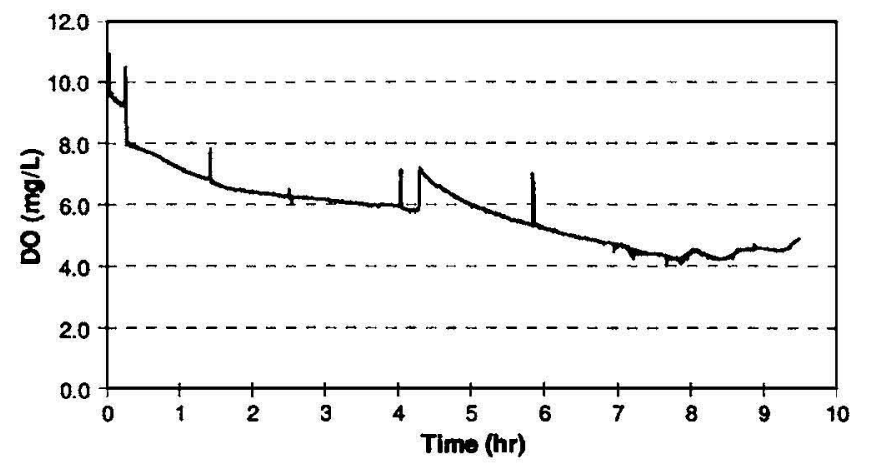

Fig. 4. Dissolved oxygen concentration from one microreactor using the ML 357 carrier. The microreactor was charged with fresh glucose media at time 0 and $4.3 \mathrm{~h}$. The supersaturation observed at the start of the experiment is attributed to interference from carriers and air bubbles trapped on the surface of the oxygen probe.

About three to five weeks were needed for a quasi-steady state biofilm to be achieved, as measured by the substrate consumption rate. The substrate consumption rates for Kaldnes Carrier Element-Original (KA-O) are shown in Fig. 5 for a series of five days, starting five weeks postinoculation. From this figure one observes that the rates of afternoon experiments were significantly higher than morning experiments of the same day. This trend was observed with all carriers tested [Fig. 6(a)], with the afternoon substrate consumption rates $63-138 \%$ higher than the morning rates for each carrier. Morning results are reflective of a "starved" biofilm as the microreactors had sat dormant the previous night. Afternoon results are reflective of an "adapted" biofilm with potential activity contributions from biofilm growth on other surfaces inside the microreactor.

To determine the primary cause of the higher afternoon measurements (i.e., adaptation versus wall growth of biofilm) three batch experiments were conducted on the same day. All carriers were placed in clean and sterile flasks immediately prior to each substrate consumption rate measurement. The biofilms on the carriers were cropped by subjecting them to a $10-\mathrm{s}$ vortex as described in the "Materials and Methods" section. The results in Fig. 6(b) demonstrate a significant increase in rate from morning

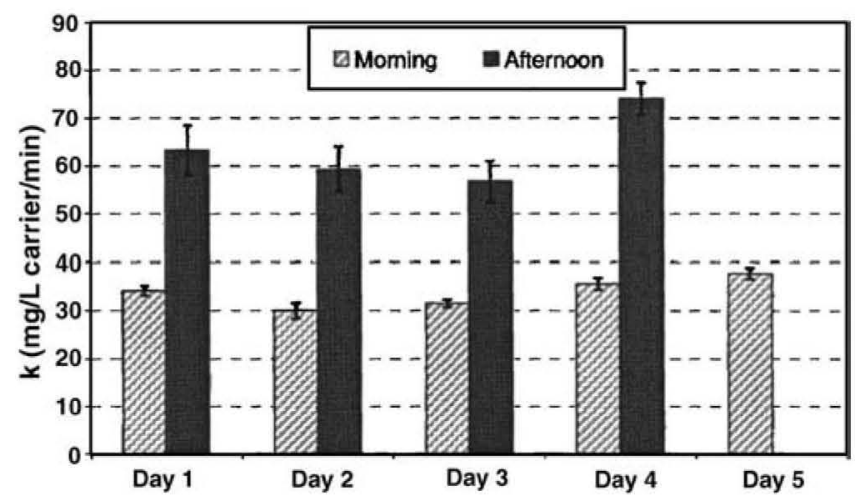

Fig. 5. The substrate consumption rates for KA-O as a function of time. Clean flasks were used in the morning experiment and flasks were not changed for the afternoon experiments. Approximate time for morning and afternoon experiments is 9:30 and 13:30, respectively. Mean values and $95 \%$ confidence intervals are shown $(n=3)$. 

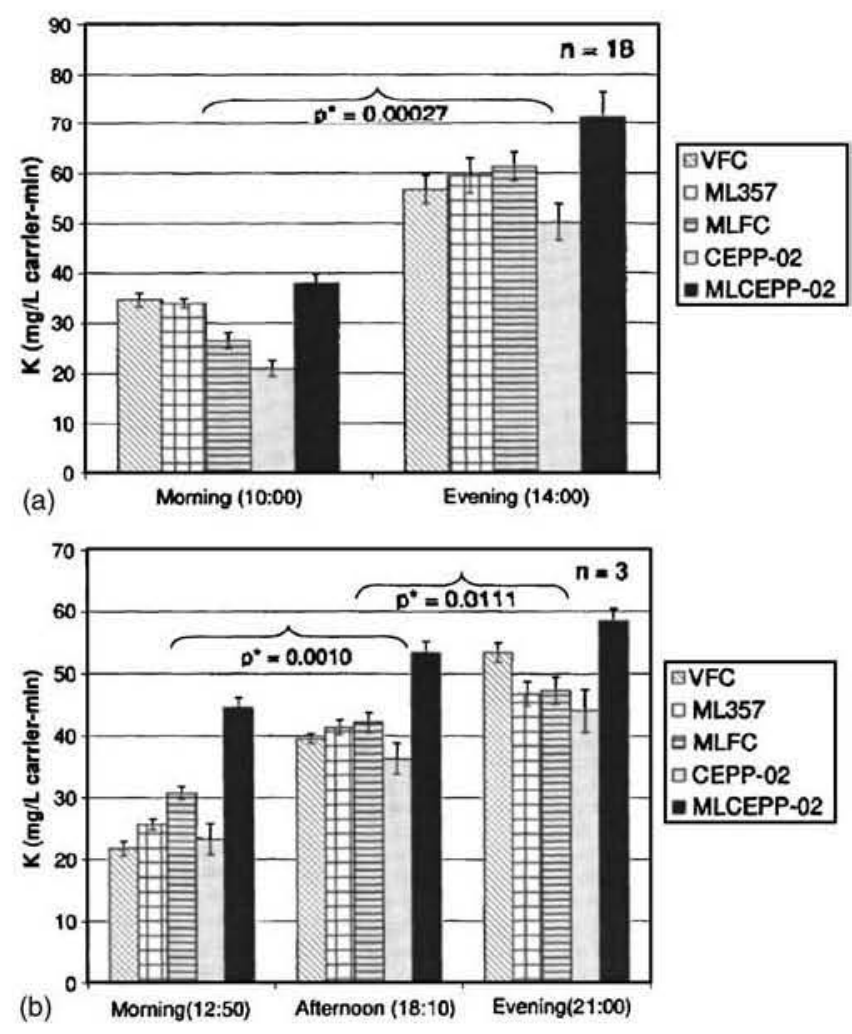

Fig. 6. (a) Substrate consumption rates measured in the morning (10:00) and afternoon (14:00) using same flasks without cleaning flasks between measurements. Mean values and $95 \%$ confidence intervals are shown. Paired t-test was done using $\alpha=0.05$. (b) Reaction rates measured three times in a single day using new flasks each time. Mean values and $95 \%$ confidence intervals are shown. Paired t-test was done using $\alpha=0.05$. to afternoon and again to evening, indicating that the cells were adapting to the presence of substrate. The increases in substrate consumption rates using the same flasks for moming and afternoon experiments [Fig. 6(a)] were usually even greater than the increase obtained from experiments using all new flasks [Fig. $6(\mathrm{~b})]$, indicating that wall growth also had a significant contribution to the rate constant measurement when clean flasks were not used.

The substrate consumption rates (using clean flasks after an overnight fast) of ten carriers are shown in Fig. 7. The moming values are used in all subsequent analyses so as to eliminate potential wall-growth contributions. Furthermore, since waste stream concentrations are highly variable and may include stagnant periods, we believe that it is preferable to make comparisons of carriers using the more conservative, nonadapted substrate consumption rates that occur in the morning. Syntrex 1220 and Kaldnes Carrier Element-Modified exhibited the highest reaction rates and Kaldnes Carrier Element-Original, Macrolite Modified CEPP-02, Macrolite 357, and Virgin Foam Cubes exhibited the next highest activities. Several factors contributing to biological activity are discussed below.

\section{Surface Roughness}

Surface roughness was determined qualitatively by direct visualization of the carriers and, in some cases, by SEM images (not shown). "Rough" surfaces are defined as carriers that had coarse, uneven surfaces due to numerous pits, pores, or small bumps on the surface. "Smooth" surfaces lacked these features. Table 1 contains this characterization of each carrier. It was observed that the accumulation of biofilm on smooth surfaces was visibly less than on rough surfaces. Surface roughness appears to promote biofilm accumulation by providing a larger surface area for attachment and by providing sheltered anchoring points that protect the biofilm from fluid shearing and collision. For example, carriers with smooth surfaces (WL, RP, CEPP-02, VPP, Fig. 7) exhibited relatively low reaction rates. Conversely Macrolite 357 and Macrolite Modified CEPP -02 , which both have relatively rough surfaces,

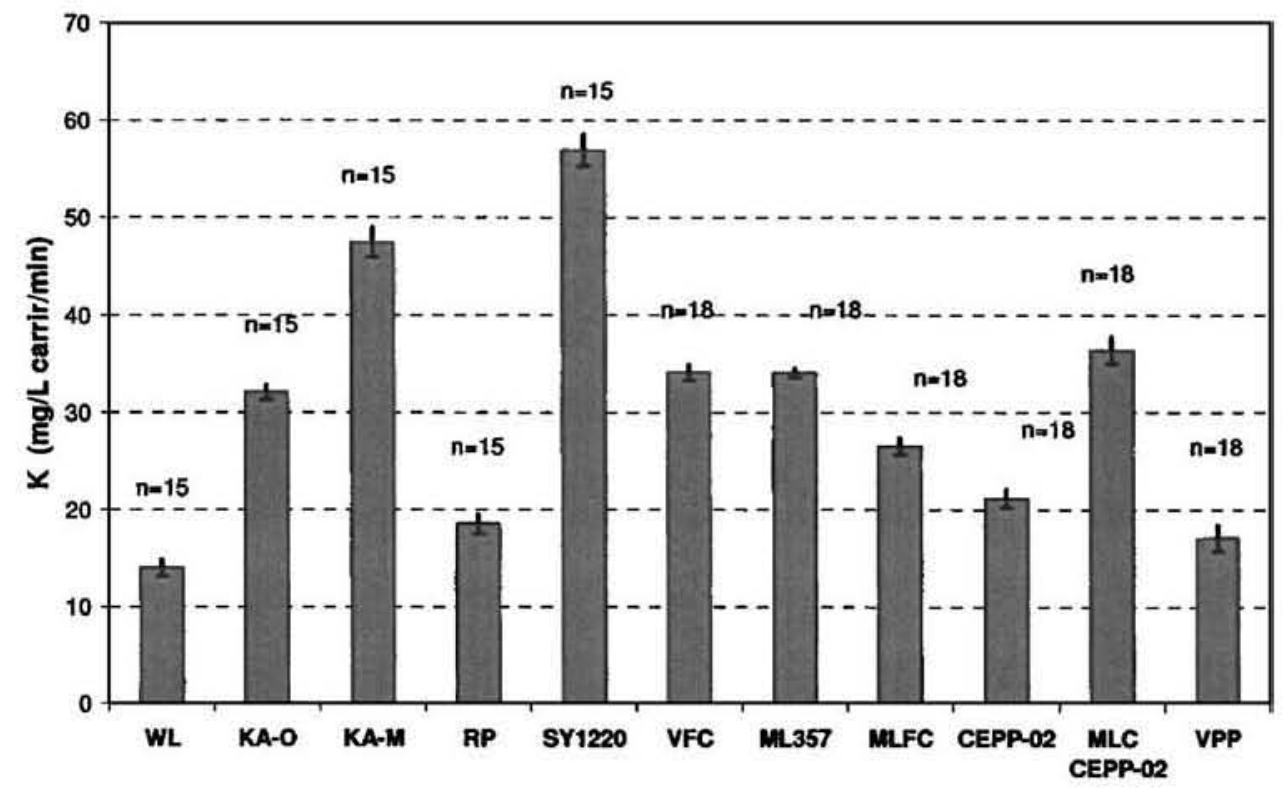

Fig. 7. Mean values of substrate consumption rates ( $\pm 95 \%$ confidence interval) during the quasi-steady state period. All measurements were performed in the morning, after an overnight fast, using clean flasks. Measurements of the 10 carriers were not performed in the same runs. 


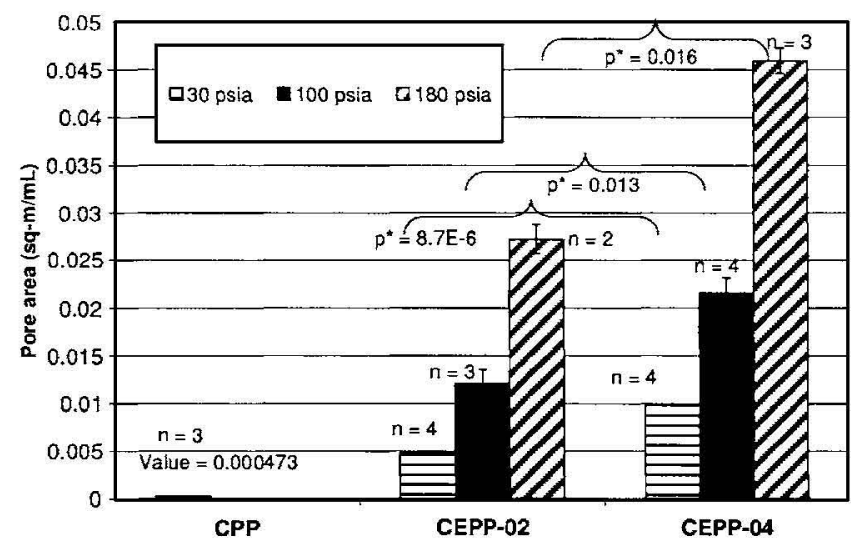

Fig. 8. Surface area accessible for biofilm growth of CEPP with different levels of expansion; mean values $\pm 95 \%$ confidence interval; t-test was done using $\alpha=0.05$

exhibited high reaction rates (Fig. 7). The use of a Macrolite fines coating, which imparts roughness to otherwise smooth materials, had mixed results. It increased the reaction rate of the relatively smooth CEPP-02 (CEPP-02 versus MLCEPP-02), while decreasing the reaction rate of the highly porous virgin foam cubes (VFC versus MLFC). The latter result may be attributed to the Macrolite coating blocking access to some pores on the VFC.

\section{Protection from Shear Forces}

Kaldnes Carrier Element-Modified (interior fins) exhibited a higher reaction rate than Kaldnes Carrier Element-Original (exterior fins) (Fig. 7), indicating that protection from fluid shear forces promotes biofilm growth.

\section{Surface Area}

Measurements of surface area, as quantified by mercury porosimetry, are shown in Fig. 8 for the chopped expanded polypropylene carriers with varying degrees of expansion. At 30 psia, the reported surface area corresponds to the cumulative area of all pores greater than $6.25 \mu \mathrm{m}$ in diameter; 100 psia corresponds to the cumulative area of all pores greater than $1.75 \mu \mathrm{m} ; 180$ psia corresponds to the cumulative area of all pores greater than $1 \mu \mathrm{m}$ (assuming the compressive effects on the granule are negligible). As expected, the surface area increases with the degree of expansion, over all ranges of pore sizes. The substrate consumption rate also increases with degree of expansion (Fig. 9), most likely resulting from the corresponding increase in surface area. There was no significant difference between the activities of CEPP-04 and CEPP-06. This trend is further corroborated by the dry weight biomass measurements depicted in Fig. 10. Specific surface area measurements using nitrogen adsorption on CEPP-04, SY1220, and MLCEPP-02 yielded values of 35,67 , and $82 \mathrm{~m}^{2} / \mathrm{L}$, respectively. These values directly correlated with their respective carriers' glucose consumption rates given in Figs. 11 and 12 (32, 48, and $52 \mathrm{mg} / \mathrm{L} / \mathrm{min}$, respectively). However, the glucose consumption rate in terms of surface area was not uniform across the three carriers $\left(0.91,0.72\right.$, and $0.64 \mathrm{mg} / \mathrm{m}^{2} / \mathrm{min}$ for CEPP-04, SY1220, and MLCEPP-02, respectively), indicating that surface area alone was not the sole determinant of biological activity, and that other factors such as pore size, surface charge, and surface roughness may play important roles in biofilm development.

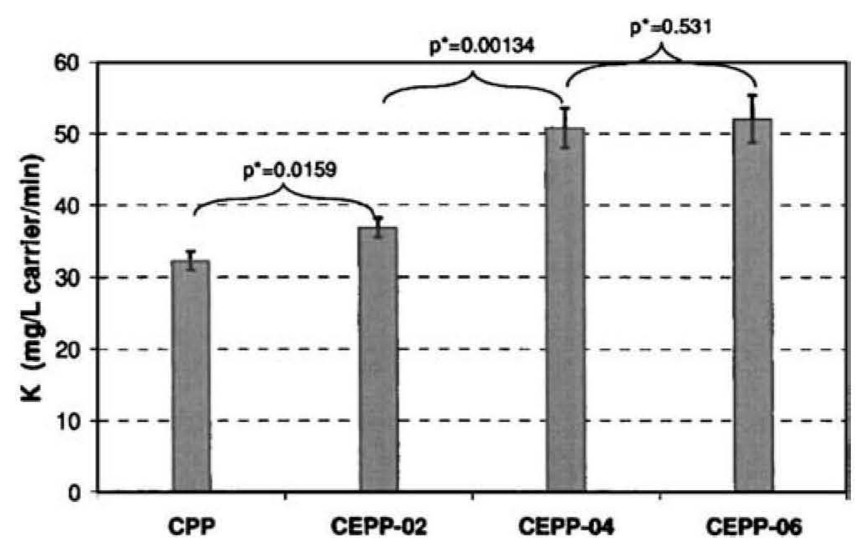

Fig. 9. Substrate consumption rates of chopped expanded polypropylene carriers made with four levels of expansion: none (CPP), low (CEPP-02), moderate (CEPP-04), and high (CEPP-06). To ensure that each microreactor had the same degree of adaptation, three experiments were performed consecutively in one day, and new flasks were used for each experiment. The rate constants from only the third experiment (after adaptation) are shown. Mean values of rate constants ( $\pm 95 \%$ confidence interval, $n=12)$ during the steady state stage; t-test was done using $\alpha=0.05$.

\section{Substrate Type}

The steady state substrate consumption rates were measured for the three top-performing carriers (SY1220, KA-M, and CEPP-04) in both glucose- and molasses-containing media (Fig. 11). Not surprisingly, given the complex carbohydrate structure of molasses compared to simple glucose, the substrate consumption rates were lower in the molasses compared to glucose with SY1220 and KA-M, by $17 \%$ and $15 \%$ respectively. The larger carbohydrate molecule in the molasses media has a slower transport rate than glucose within the biofilm, and thus supports a thinner biofilm as evidenced by the lower substrate consumption rate. The CEPP-04 substrate consumption rates were nearly identical in both media. Since the CEPP-04 glucose consumption rate was already lower than that of the other two carriers, this indicates

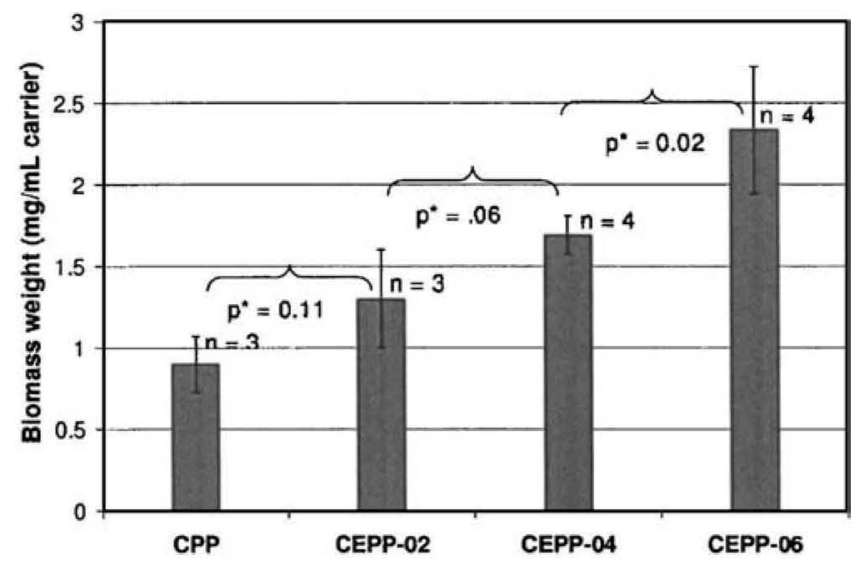

Fig. 10. Dry weight measurements of CEPP with different levels of expansion, measured at the conclusion of the experiment described in Fig. 9; mean values $95 \%$ confidence interval; $\mathbf{t}$-test was done using $\alpha=0.05$ 


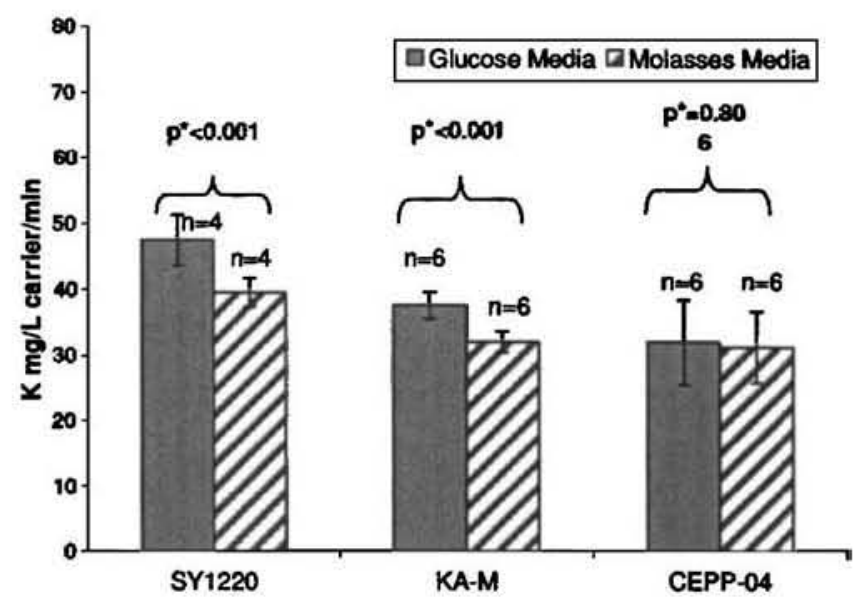

Fig. 11. Comparison of substrate consumption rates glucose and molasses media in microreactor experiments. Experiments were conducted in the same shaker bath test. Rate constants in molasses media were calculated from COD measurements. Mean values \pm standard deviation from both $\alpha=0.05$.

that the limiting factor for biofilm growth on the CEPP-04 was possibly another variable (i.e., oxygen transport or available surface area) rather than substrate transport.

\section{Scale-up}

Substrate consumption rates in microreactors and the bioreactor are given in Fig. 12 for three carriers. Glucose consumption rate was $27-29 \%$ lower in the bioreactor compared to the microreactors for MLCEPP-02 and KA-M, while it was $43 \%$ greater in the bioreactor with ML357.

\section{Discussion and Conclusions}

The procedure described here provides a detailed method for the economical evaluation of biofilm carriers that yields objective and insightful data for quantifying biofilm activity. Zero-order reaction kinetics adequately represented the glucose consumption rate and allowed the biofilm activity to be represented by a single parameter. After three weeks, a quasi-steady state was achieved as

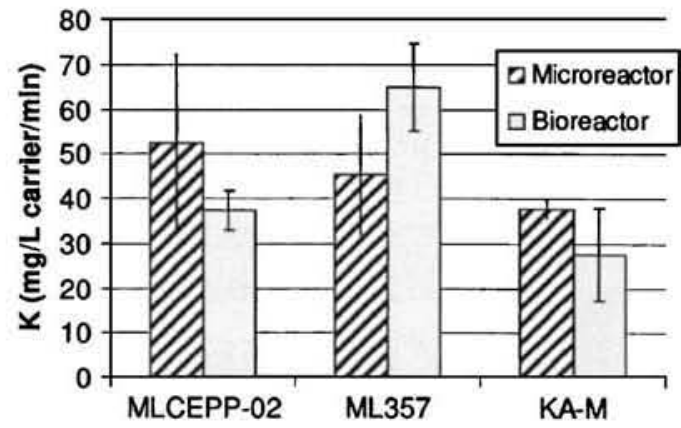

Fig. 12. Comparison of rate constants in 3-L batch reactor and microreactors, using glucose media. Rate constants were calculated from measurements from both morning and afternoon experiments, using both clean and dirty flasks, for both the microreactor and bioreactor experiments. Mean values \pm standard deviation; $t$-test was done using $\alpha=0.05$. characterized by oscillations in glucose consumption rates that were regular (on a daily period) and reproducible. Oscillations in biofilm thickness have been observed previously, and are usually attributed to repeated cycles of biofilm growth and die-off caused by oxygen limitation. Since the carriers in this work were rinsed and vigorously mixed between moming and afternoon measurements, it is unlikely that the twofold increase in rates in the aftemoon were caused by a doubling in biofilm thickness. In the work presented here it was determined that adaptation to a glucose-rich environment, after a quiescent period during overnight starvation, was the primary determinant of the daily oscillations in glucose consumption. Biofilm growth on surfaces within the microreactors was also shown to contribute to the substrate consumption rate when measurements were done using flasks that were not cleaned immediately prior to the beginning of the experiment. These results indicate that in order to meaningfully compare substrate consumption rates of different carriers, the time since feeding must be the same for each carrier and measurements must be done using clean flasks.

The characteristics of surface roughness and surface area appear to have the biggest impact on biological activity based on the results presented here. Rough coatings such as Macrolite fines can increase the substrate consumption rates of smooth carriers, while decreasing the consumption rates of carriers that already have highly porous surfaces. The Macrolite fine coating was observed to fill in the pores, thus reducing surface coarseness. The substrate consumption rate was positively correlated with dry biomass and with measurements of surface area. If all the substrate consumption rate data were presented in terms of surface area of the carriers, rather than bulk volume of the carriers, the interpretation of the results as to the most effective carrier would be very different. However, at the industrial scale the carriers are sold and priced by bulk volume, and thus volume is a more meaningful basis of comparison. Furthermore, use of bulk volume as the rate basis is more meaningful in terms of estimating full scale reactor size and full scale economics.

Although substrate consumption rates can be correlated with physical characteristics, such as roughness and surface area, the actual difference among the carriers in terms of substrate consumption rates may be fundamentally caused by the accessibility of the carrier surface area to oxygen, which is usually considered to be the substance limiting for cell growth. In both the microreactors and the bioreactor the oxygen concentration was always maintained at about $50 \%$ saturation in the bulk fluid. We recognize that even at this saturation level, it is likely that the inner surface of the biofilm on some of the carriers may be oxygen limited, and thus the differences in substrate consumption rate observed among the carriers in the microreactor experiments may be directly attributed to differences in oxygen availability at the inner layer of the biofilm. Thus, carriers that are relatively nonporous yet still exhibit a high degree of surface roughness (e.g., SY 1220) may be able to support a greater surface area of biofilm, but with a diminished thickness that permits full oxygen penetration, resulting in an aerobically efficient biofilm volume. Conversely, carriers that have relatively large and deep pores (e.g., CEPP-04), would have thicker biofilms that would create oxygen limiting conditions, and therefore have a less aerobically efficient biofilm volume. This aerobically efficient biofilm volume is essentially evaluated by the overall glucose consumption rate.

It is recognized that the actual industrial environment will possess a mixture of complex carbohydrate sources, and will exist in a more complex bioreactor process. Relatively small changes in substrate consumption rates $(<17 \%)$ were obtained when the 
glucose was replaced with the polysaccharide molasses, indicating that a screening method using the more economical glucose is at least a reasonable substitute for tests with actual wastewater during the initial screening process. Glucose is a favorable choice for screening since glucose analysis is essentially instantaneous (compared to the minimum of two hours needed for COD) and the cost per sample is considerably lower than that of COD. Carriers that achieve the greatest glucose substrate consumption rates in the microreactor tests will require testing with actual wastewater to determine realistic substrate consumption rates as well as to identify the optimum carrier for a particular wastewater.

Substrate consumption rates in the 3-L bioreactor were from $27 \%$ lower to $43 \%$ greater than those measured with the same carriers in microreactors. This large variation is not unexpected considering the significant differences in mixing that occur during scale-up. More experiments will need to be conducted with different carriers in the 3-L (or larger) bioreactor to evaluate the ability of the microreactor system to predict carrier effectiveness in large-scale systems.

The conclusions reached during this study are based on glucose consumption rates measured in a simple media with glucose as the sole carbon and energy source. The method is relatively simple, economical to implement, and y ields reproducible results. Although the method requires approximately four weeks duration, numerous carriers can be evaluated simultaneously with multiple repetitions of substrate consumption rate measurements. Thus, this approach is more economical than successive evaluations in a pilot-scale reactor. Methods to ensure a similar biofilm ecology among the different carriers were presented. The importance of conducting measurements at the same time postrefeeding, because of the depression of activity caused by overnight starvation, was demonstrated. The contribution of wall-growth to biofilm activity was shown to be significant and must also be considered in the experimental design. This method should find usefulness as a tool to rapidly and economically screen newly designed carriers for their potential effectiveness in wastewater bioreactor systems utilizing fluidized bed, moving bed, or other aerated biological filter bed technologies.

\section{Acknowledgments}

Funding from Kinetico, Inc., to support this research is gratefully acknowledged. The writers would also like to thank Upah Tech, Inc, for their input with microbiological ecology.

\section{References}

Araki, N., Ohashi, A., Machdar, I., and Harada, H. (1999). "Behaviots of nitrifiers in a novel biofilm reactor employing hanging sponge-cubes as attachment site." Water Sci. Technol 39(7), 23-31.

Becker, K. (1998). "Detachment studies on microfouling in natural biofilms on substrata with different surface tensions." Int. Biodeter. Biodegrad., 41(1), 93-100.

Belgiono, V., De Feo, G., and Napoli, R. (2003). "Combined carbonaceous removal and nitrification with biological aerated filtets." $J$. Environ. Sci. Health, Part A: Toxic/Hazard. Subst. Environ. Eng., $38(10), 2147-2156$.

Bos, R., van der Mei, H. C., Gold, J., and Busscher, H. J. (2000). "Retention of bacteria on a substratum surface with micro-pattemed hydophobicity." FEMS Microbiol. Lett. 189(2), 311--315.

Breitenbucher, K., Siegl, M., Knupfer, A., and Radke, M. (1990). "Openpore sintered glass as a high efficiency support medium in bioreactors-new results and long-term experiences achieved in high rate anaerobic digestion." Water Sci. Technol, 22(1--2), 25-32.

Brown, D. A., Beveridge, T. J., Keevil, C. W., and Sheriff, B. L. (1998), "Evaluation of microscopic techniques to observe iron precipitation in a natural microbial biofilm." FEMS Microbiology Ecology, 26i(4), $297-310$.

Chandravathanam, S., and Murthy, D. (1999). "Studies in nitrification of municipal sewage in an upfiow biofilter." Bioprocess Eng., 21(2), $117-122$.

Davey, M. E., and O'Toole, G. A. (2000). "Microbial biofilms from ecology to moleculat genetics." Microbiol Mol Biol Rev, 64(4), 847867.

Fletcher, M. (1991). "The physiological activity of bacteria attached to solid surfaces." Adv. Microb. Physiol, 32, 53-85.

Flint, S. H., Brooks, J. D., and Btemer, P. J. (1997). "The influence of cell surface properties of thermophilic streptococci on attachment to stainless steel." J. Appl. Microbiol., 83(4), 508-517.

Gilbert, E. S., Khlebrikov, A., Meyer-llse, W., and Keasling, J. D. (1999). "Use of soft $\mathrm{x}$-ray microscopy for analysis of early-stage biofilm formations." Water Sci. Technol, 39(7), 269-272.

Jones, D. S., Adair, C. G., Mawhinney, M., and Gorman, S. P. (1996). "Standardization and comparison of methods employed for microbial cell surface hydrophobicity and charge determination." Int. J. Pharm, $131(1), 83-89$.

Lazarova, V., and Manem, I. (1995). "Biofilm characterization and activity analysis in water and wastewater treatment." Water Res. 29(10), $2227-2245$

Münch, E., Barr, K., Watts, S., and Keller, J. (2000). "Suspended carrier technology allows upgrading high-rate activated sludge plants for nitrogen removal via process intensification." Water Sci. Technol. 41(45), 5-12.

Nation, I. L. (1983). "A new method using hexamethyldisilazzne for preparation of soft tissues for scanring electron microscopy." Stain Technol. 58(6), 347-351.

$\emptyset$ degaard, H. (2000). "Advanced compact wastewater treatment based on coagulation and moving bed biofilm process." Water Sci. Technol., $42(12), 33-48$.

Okkerse, W. J. H., Ottengraf, S. P. P., and Osinga-Kuipers, B. (2000). "Biofilm thickness variability investigated with a laser triangulation sensor." Biotechnol. Bioeng., 70(6), 619-629.

Payraudeau, M., Pearce, A., Goldsmith, R., Bigot, B., and Wicquart, F. (2001). "Experience with an up-flow biological aerated filter (BAF) for tertiary treatment: From pilot trials to full scale implications." Water Sci Technol $44(2-3), 63-68$.

Rodgers, M., and Zhan, X.-M. (2004). "Biological nitrogen removal using a vertically moving biofilm system." Bioresour. Technol. 93(3), 313-319.

Salkinoja-Salonen, M. S., Hakulinen, R., Valo, R., and Apajalahti, J. (1983). "Biodegradation of recalcitrant organochlorine compounds in fixed film reactors." Water Sci. Technol, 15, 309-319.

Sanford, B. A., Thomas, V. L., Mattingly, S. U., Rarnsay, M. A., and Miller, M. M. (1995). "Lectin-biotin assay for slime present in in situ biofilm produced by Staphylococcus epidermidis using transmission electron mictoscopy (TEM)." J. Ind. Microbiol., 15(3), 156-161.

Shreve, G. S., Olsen, R. H., and Vogl, T. M. (1991). "Development of pure culture biofilms of $P$. putida on solid supports." Biotechnol. Bioeng., 37(6), 512 518.

Teixeira, P., and Oliveira, R. (1998). "The importance of surface properties in the selection of supports for nitrification in airlift bioreactors." Bioprocess Eng., 19(2), 143-147.

Van Loosdrecht, M. C. M., Eikelboom, D., Gjaltema, A., Mulder, A., Tijhuis, L., and Heijnen, J. J. (1995). "Biofilm structures." Water Sci. Technol., 32(8), 35-43.

Wimpenny, I., Manz, W., and Szewzyk, U. (2000). "Heterogeneity in biofilms." FEMS Microbiol. Rev, 24(5), 661-671.

Yoo, I., and Kim, D. (2001). "Effects of hydraulic backwash load on effluent quality of upflow BAF." J. Environ. Sci. Health, Part A: Toxic/Hazard. Subst. Environ. Eng., 36(4), 575-585. 